\title{
The Effect of a Metal Wall on Confinement in JET and ASDEX Upgrade
}

\author{
M.N.A. Beurskens ${ }^{1}$, J. Schweinzer ${ }^{2}$, C. Angioni ${ }^{2}$, A. Burckhart ${ }^{2}$, C.D. Challis ${ }^{1}$, I. Chapman ${ }^{1}$, \\ R. Fischer ${ }^{2}$, J. Flanagan 1 , L. Frassinetti ${ }^{3}$, C. Giroud ${ }^{1}$, J Hobirk ${ }^{2}$, E. Joffrin ${ }^{4}$, A Kallenbach ${ }^{2}$, \\ M. Kempenaars ${ }^{1}$, M. Leyland ${ }^{5}$, P. Lomas ${ }^{1}$, G. Maddison ${ }^{1}$, M Maslov ${ }^{1}$, R. McDermott ${ }^{2}$, \\ R. Neu ${ }^{2}$, I. Nunes ${ }^{6}$, T. Osborne ${ }^{7}$, F. Ryter ${ }^{2}$, S. Saarelma ${ }^{1}$, P.A. Schneider ${ }^{2}$, P. Snyder ${ }^{7}$, G \\ Tardini $^{2}$, \\ E. Viezzer ${ }^{2}$, E. Wolfrum² ${ }^{2}$, the ASDEX Upgrade Team, and JET-EFDA Contributors*
}

JET-EFDA, Culham Science Centre, Abingdon, OX14 3DB, UK.

${ }^{I}$ EURATOM /CCFE Fusion Association, Culham Science Centre, Abingdon, OX14 3DB, UK.

${ }^{2}$ Max-Planck-Institut für Plasmaphysik, EURATOM Association, D-85748 Garching, Germany.

${ }^{3}$ Div. of Fusion Plasma Physics, Association EURATOM-VR, KTH, SE-10044 Stockholm, Sweden .

${ }^{4}$ Association Euratom-CEA, IRFM, F-13108 St-Paul-Lez-Durance, France.

${ }^{5}$ York Plasma Institute, Department of Physics, University of York, Heslington, York, YO10 5DD, UK.

${ }^{6}$ Instituto de Plasmas e Fusão Nuclear, Associação EURATOM-IST, Lisboa, Portugal .

${ }^{7}$ General Atomics, PO Box 85608, San Diego, California 92186-5608, USA .

* See the Appendix of F. Romanelli et al., Proceedings of the 24th IAEA Fusion Energy Conference

2012, San Diego, USA

Corresponding author e-mail: Marc.Beurskens@ccfe.ac.uk

\begin{abstract}
In both JET and ASDEX Upgrade (AUG) the plasma energy confinement has been affected by the presence of a metal wall by the requirement of increased gas-fuelling to avoid tungsten pollution of the plasma. In JET with a beryllium/tungsten wall the high triangularity baseline H-mode scenario (i.e. similar to the ITER reference scenario) has been strongest affected and the benefit of high shaping to give good normalised confinement of $\mathrm{H}_{98} \sim 1$ at high Greenwald density fraction of $\mathrm{f}_{\mathrm{GW}} \sim 0.8$ has disappeared. In AUG with a full tungsten wall a good normalised confinement $\mathrm{H}_{98} \sim 1$ could be achieved in the high triangularity baseline plasmas, albeit at elevated normalised pressure $\beta_{\mathrm{N}}>2$. The confinement lost with respect to the carbon devices can be largely recovered by the seeding of nitrogen in both JET and AUG. This suggests that the absence of carbon in JET and AUG with a metal wall may have affected the achievable confinement. Three mechanisms have been tested that could explain the effect of carbon or nitrogen (and the absence thereof) on the plasma confinement. First it has been seen in experiments and by means of non-linear gyrokinetic simulations (with the GENE code), that nitrogen seeding does not significantly change the core temperature profile peaking and does not affect the critical ion temperature gradient. Secondly, the dilution of the edge ion density by the injection of nitrogen is not sufficient to explain the plasma temperature and pressure rise. For this latter mechanism to explain the confinement improvement with nitrogen seeding, strongly hollow $Z_{\text {eff }}$ profiles would be required, which is not supported by experimental observations. The confinement improvement with nitrogen seeding cannot be explained with these two mechanisms. Thirdly, detailed pedestal structure analysis in JET high triangularity baseline plasmas have shown that fuelling of either deuterium or nitrogen widens the pressure pedestal. However, this only leads to a confinement benefit in the case of nitrogen seeding where as the pedestal widens, the obtained pedestal pressure gradient is conserved. In the case of deuterium fuelling the pressure gradient is strongly degraded in the fuelling scan leading to no net confinement gain due to the pedestal widening. The pedestal code EPED correctly predicts the pedestal pressure of the unseeded plasmas within $+/-5 \%$, however it does not capture the complex variation of pedestal width and gradient with fuelling and impurity seeding. Also it does not predict the observed increase of pedestal pressure by nitrogen seeding. Ideal peeling ballooning MHD stability analysis shows that the widening of the pedestal leads to a down shift of the marginal stability boundary by only $10-20 \%$. However the variations in the pressure gradient observed in the experiment is much larger and spans a factor of more than two. As a result the experimental points move from deeply unstable to deeply stable on the stability diagram in a deuterium fuelling scan. In AUG-W nitrogen seeded plasmas, also a widening of the pedestal has been observed, consistent with the JET observations. The absence of carbon can thus affect the pedestal structure, and mainly the achieved pedestal gradient, which can be recovered by seeding nitrogen. The underlying physics mechanism is still under investigation and requires further understanding of the role of impurities on the pedestal stability and pedestal structure formation.
\end{abstract}




\section{Introduction}

The confinement in tokamak plasmas can be affected by the choice of first wall materials due to plasma impurity composition and measures required to prevent first wall damage. The understanding of these factors is crucial for an accurate estimate of the plasma performance in future fusion devices such as ITER and DEMO where the use of a carbon first wall, common in present devices, is prohibited because of the high tritium-fuel retention properties of carbon [1]. For this reason the ASDEX Upgrade (AUG) carbon wall (AUG-C) was replaced in a step wise manner from 1999 to 2007 by a DEMO-relevant full W-wall and divertor (AUG-W) [2], whereas in JET the carbon wall (JET-C) was fully replaced in 20092010 by an ITER-like Be main chamber first wall and W divertor (JET-ILW) [3].

This paper discusses the impact of these first-wall changes on plasma confinement in the AUG and JET tokamaks. We have found a common pattern in these full metal devices that the requirement to screen high- $Z$ impurity fluxes by means of increased gas fuelling has adversely affected the pedestal and core confinement. In this paper the main focus is on the ITER baseline $\mathrm{H}$-mode scenario at $\mathrm{I}_{\mathrm{p}}=15 \mathrm{MA}$ and $\mathrm{B}_{\mathrm{t}}=5.3 \mathrm{~T}, \beta_{\mathrm{N}}=1.8$, plasma triangularity $\delta_{\mathrm{av}} \approx$ 0.4 with target fusion product of $\mathrm{Q}=\mathrm{P}_{\mathrm{D}-\mathrm{T}} / \mathrm{P}_{\text {in }}=10$. The baseline ELMy H-mode development has been a main line of research in JET over the past decades [e.g. 4]. In JET-C baseline ELMy H-mode plasmas achieved good normalized confinement with the normalised confinement following the ITER physics base scaling [5] of $\mathrm{H}_{98(\mathrm{y}, 2)} \approx 1$ in un-fuelled plasmas [6]. Moreover, in the high triangularity configuration $\left(\delta_{\mathrm{av}} \approx 0.4\right)$, good normalised confinement could be maintained up to a plasma density approaching the Greenwald density fraction $\left(\mathrm{n} / \mathrm{n}_{\mathrm{gw}} \approx 1.0\right)$ with elevated fuelling levels (2 to $310^{22}$ electrons/s) [4, 6]. A similar observation has been made for AUG in [7]. The good normalised confinement in the JET-C plasmas is maintained through a widening of the edge pedestal as the fuelling level is increased [8], where a steep pedestal pressure gradient could be maintained over a wider region, resulting in an increase of the pedestal-top pressure. However, at low plasma triangularity $\left(\delta_{\mathrm{av}} \approx 0.2\right)$, the global confinement was generally degraded at increasing fuelling levels [4]. Unfortunately, the pedestal in the JET-C low triangularity plasmas could not be monitored due to diagnostic constraints. In JET-C an extensive nitrogen seeding experiment was conducted in high triangularity baseline H-mode plasmas in view of divertor heat-load mitigation requirements in JET-ILW [9]. The $\mathrm{N}_{2}$ seeding did not improve confinement in these JET-C H-mode plasmas. However, when the experiment was repeated in the same plasma configuration in JET-ILW an advantageous effect was found on the plasma confinement $[10,11,12]$.

In AUG the main focus of plasma scenario development has been on the so-called improved H-mode scenario [13,14,15]. In AUG-C the improved H-mode plasmas could sustain good normalised confinement at low plasma density $\left(\mathrm{n} / \mathrm{n}_{\mathrm{gw}}=0.4-0.5\right)$ with $\mathrm{H}_{98(\mathrm{y}, 2)} \sim$ 1.4. After the installation of the full tungsten wall the required increased fuelling led to operation at higher plasma density $\left(\mathrm{n} / \mathrm{n}_{\mathrm{gw}}=0.6-0.8\right)$ and a reduction in normalised confinement to $\mathrm{H} 98(\mathrm{y}, 2) \sim 1.1-1.2$, but this loss in confinement could be partly recovered by Nitrogen seeding [15]. The nitrogen seeded improved H-mode plasmas again approached a normalised confinement of $\mathrm{H}_{98(\mathrm{y}, 2)} \sim 1.3$. This improved confinement compared to the unseeded plasmas was thought to be due to a so-called dilution effect which requires hollow $Z_{\text {eff }}$ profiles [15]. In addition, in a straight comparison of two plasmas with and without nitrogen seeding, a broader pedestal was observed when nitrogen was seeded.

In addition to the improved H-mode plasmas, the development of the baseline H-mode scenario has now regained interest at AUG in view of the importance of this scenario for ITER. In this paper we will refer to recent baseline H-mode experiments, but we will also 
make use of the much wider database of improved H-mode plasmas in AUG to illustrate the effect the metal wall on confinement.

The paper is divided in 5 sections. Section 2 describes the confinement changes in AUG and JET due to the presence of the metal wall; $\mathrm{W}$ in AUG-W and Be/W in JET-ILW, where the need for increased fuelling has an adverse effect on the global confinement in both devices. Section 3 will discuss the impact of the absence of carbon on the confinement in both devices and that re-introducing a carbon-like impurity (nitrogen) can have a beneficial effect on the global confinement in both devices. Finally, section 4 will discuss the possible physics mechanisms that lead to the confinement degradation with metal wall, and the (part) recovery of confinement with nitrogen seeding. Section 4.1 will discuss the role of light impurities on core turbulence suppression, and hence the impact on core profile peaking. Section 4.2 will discuss the role of edge ion-dilution, where an increased edge $Z_{\text {eff }}$ and hence reduced pedestal ion density can lead to an increased pedestal temperature. In case of a hollow $Z_{\text {eff }}$ profile this can lead to increased core pressure in the presence of temperature profile stiffness. Section 4.3 will then show the role of the pedestal structure and confinement in devices with a metal wall and will discuss the impact of impurity seeding on the edge stability. The paper is closed with discussion and conclusions in section 5 .

\section{Global confinement changes due to the presence of a metal wall}

The plasma normalised confinement in baseline H-Modes in AUG-C and AUG-W with $\mathrm{q}_{95}=3$ and a division of low and high triangularity is shown in Figure 1. The accessible density range in AUG-W is clearly restricted to higher densities (Figure 1a) due to the necessity of a nonzero gas-puff to avoid too large $\mathrm{W}$-influx. No low triangularity baseline $\mathrm{H}$-mode plasmas $\left(\delta_{\mathrm{av}} \approx 0.2\right)$ have yet been conducted in AUG-W, but across the AUG-C and AUG-W database $\mathrm{H}_{98(\mathrm{y}, 2)}$ can be sustained at higher levels for the high triangularity $\left(\delta_{\mathrm{av}} \approx 0.3-0.36\right)$ compared to the AUG-C low triangularity plasmas for a given Greenwald density fraction [16]. In AUG also a negative correlation is found between the divertor neutral background pressure and normalised confinement in both AUG-C and AUG-W as can be seen in Figure $1 \mathrm{~b}$. Nevertheless, Figure 1 shows that when the same density and the same neutral background pressure is obtained the normalised confinement in terms of $\mathrm{H}_{98 \mathrm{y}, 2}$ is similar between AUG-C and AUG-W high triangularity plasmas. For the improved $\mathrm{H}$-mode plasmas the confinement was stronger affected by the presence of the metal wall as was reported in [15].

In comparison, Figure 2 shows the confinement for JET low and high triangularity H-mode plasmas $\left(\mathrm{I}_{\mathrm{P}}=2.4-2.6 \mathrm{MA}, \mathrm{B}_{\mathrm{T}}=2.5-2.7 \mathrm{~T}, \delta_{\mathrm{av}}=0.2\right.$ and 0.4, $\left.\mathrm{P}_{\mathrm{NBI}}=14-17 \mathrm{MW}, 1.3<\beta_{\mathrm{N}}<1.8\right)$. For low triangularity plasmas, the downward trend of $\mathrm{H}_{98 \mathrm{y}, 2}$ with density in JET-ILW is similar as was reported for JET-C in [4]. However, in JET-ILW high- $\delta$ plasmas a more severe confinement degradation is observed; whereas in JET-C high $\delta$ plasmas could sustain good confinement $\mathrm{H}_{98 \mathrm{y}, 2} \sim 1$ up to the Greenwald density limit, Figure 2 shows that the low and high $\delta$ plasmas in JET-ILW are in the same range of density and $\mathrm{H}$-factor; the benefit of high $\delta$ seems to have disappeared $[9,10,17,18]$. This confinement loss in the high- $\delta$ plasmas can largely be recovered with nitrogen seeding as will be discussed in the next section. 

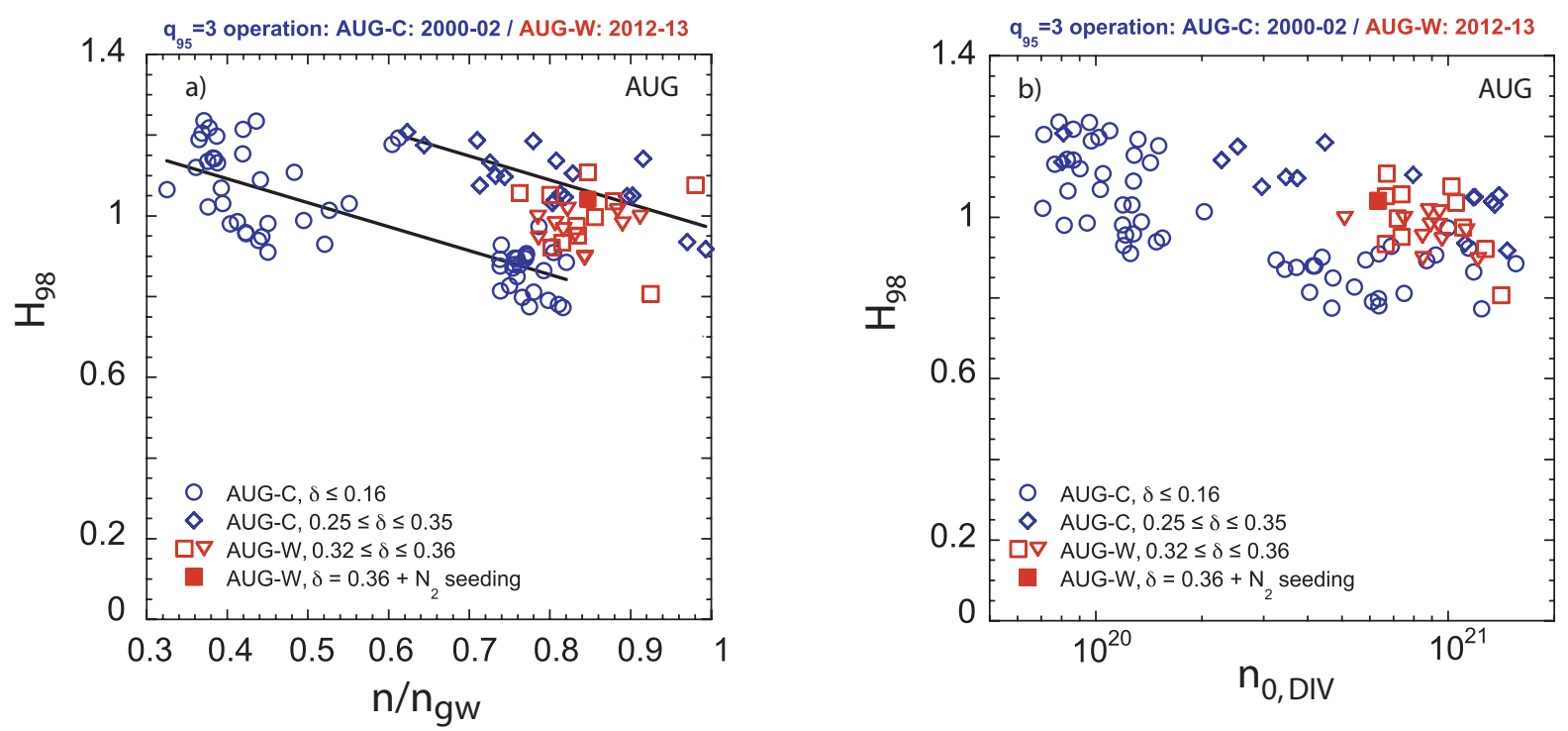

Figure 1: AUG Database comparison of normalised confinement in baseline H-modes for operation with carbon and Tungsten wall. a) $H_{98(y, 2)}$ vs line averaged density and b) $H_{98(y, 2)}$ vs neutral background pressure in the divertor. Plasma parameters: $A U G-C$ : low triangularity: $I_{p}=1 M A B_{t}=$ $2 T$, $P_{\text {tot }}$ between 4.7 - $7 \mathrm{MW}$. high triangularity: $0.6 \leq I_{p} \leq 1.2 \mathrm{MA}, 1 \leq B t \leq 2 \mathrm{~T}, 2.5 \leq P_{\text {tot }} \leq 7.5 \mathrm{MW}$ $\left(P_{N B I}=7 M W\right)$. AUG-W: high triangularity plasmas $1.1 \leq I_{p} \leq 1.2 M A .1 .8 \leq B_{t} \leq 2 T, 4.4 \leq P_{t o t} \leq 7.2$ $M W\left(P_{N B I}=3.5-6.4 M W, P_{I C R H}=0.5-0.9 M W\right.$ or $\left.P_{I C R H}=0.7-1.2 M W\right)$

The JET baseline H-mode dataset shows a strong correlation of $\mathrm{H}_{98(\mathrm{y}, 2)}$ with normalised pressure $\beta_{\mathrm{N}}$, Figure $3 \mathrm{a}$. This is a common observation in JET and has previously been reported in e.g. $[6,17,18]$. From a simple linear regression to this data-set it can be derived that $\mathrm{H}_{98(\mathrm{y}, 2)}=1$ can be achieved at $\beta_{\mathrm{N}}=1.8-1.9$. Figure $3 \mathrm{~b}$ shows that in The AUG baseline dataset shows the same trend of $\mathrm{H}_{98(\mathrm{y}, 2)}$ vs $\beta_{\mathrm{N}}$, where the AUG-W $\mathrm{H}_{98(\mathrm{y}, 2)}$ vs $\beta_{\mathrm{N}}$ data are $\sim 10 \%$ below the AUG-C fit-line. The correlation of $\mathrm{H}_{98(\mathrm{y}, 2)}$ with $\beta_{\mathrm{N}}$ would be good news for DEMO which is envisaged to operate at high $\beta_{\mathrm{N}}>2$, whereas for ITER it implies that good confinement with $\mathrm{H}_{98(\mathrm{y}, 2)}=1$ may not be obtained if the limited amount of installed input power is insufficient to reach $\beta_{\mathrm{N}}=1.8$ or above. However, it should be noted that the ITER physics base scaling [5] has a strong $\beta$ dependence of the energy confinement time as $\tau_{98(y, 2)} \sim$ $\beta^{-0.9}$. It may be that the confinement scaling of $\mathrm{H}_{98(\mathrm{y}, 2)}$ vs $\beta_{\mathrm{N}}$ in Figure 3 is due to a weaker $\beta$ dependence in the current data-sets. Dimensionless scaling experiments are planned for upcoming experimental campaign to test this dependence. 

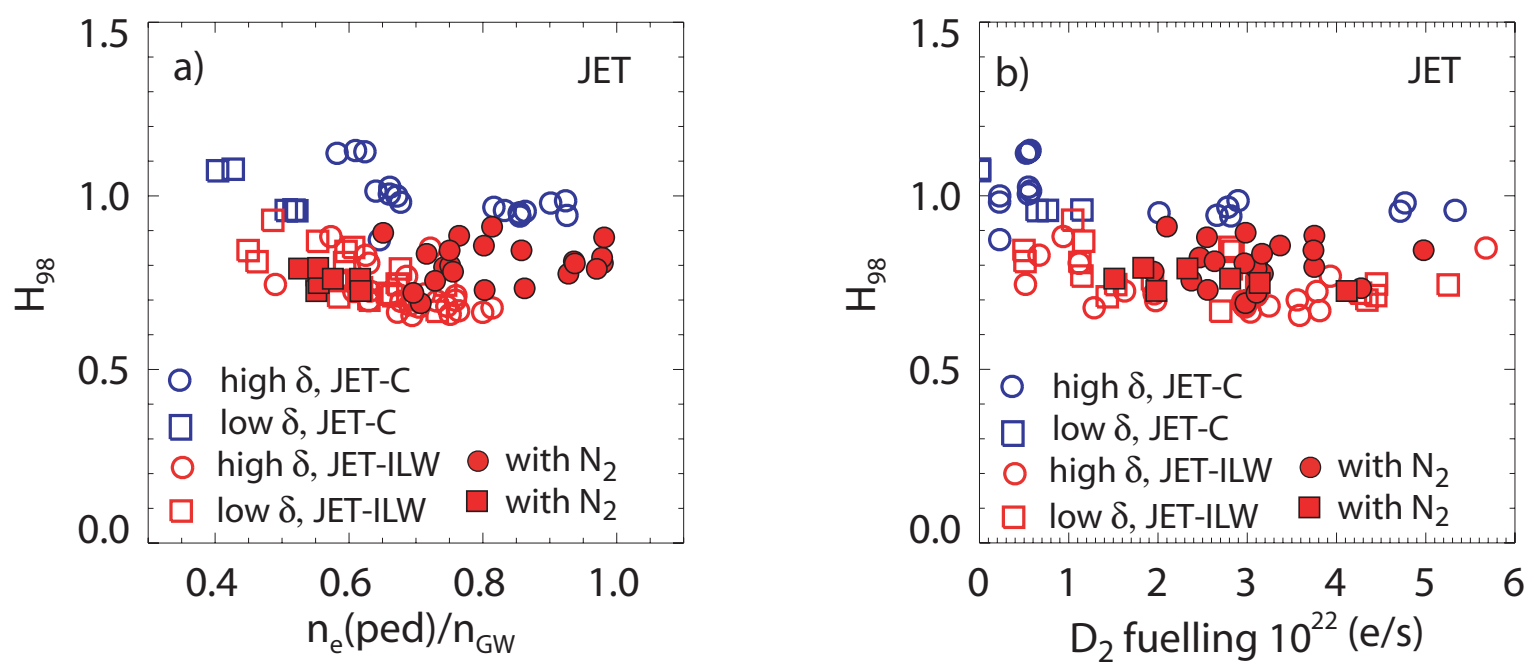

Figure 2: JET database comparison of $H_{98(y, 2)}$ for baseline Type I ELMy H-mode plasmas at low $(\delta=0.2)$ and high $(\delta=0.4)$ triangularity. $\left(I_{P}=2.5-2.7 \mathrm{MA}, B_{T}=2.3-2.7 \mathrm{~T}, P_{N B I}=14-17\right.$ $M W)$ for JET-C and JET-ILW. Open symbols are $D_{2}$ fuelling only for both JET-C and JET$I L W$, and closed symbols are with $N_{2}$ seeding in JET-ILW
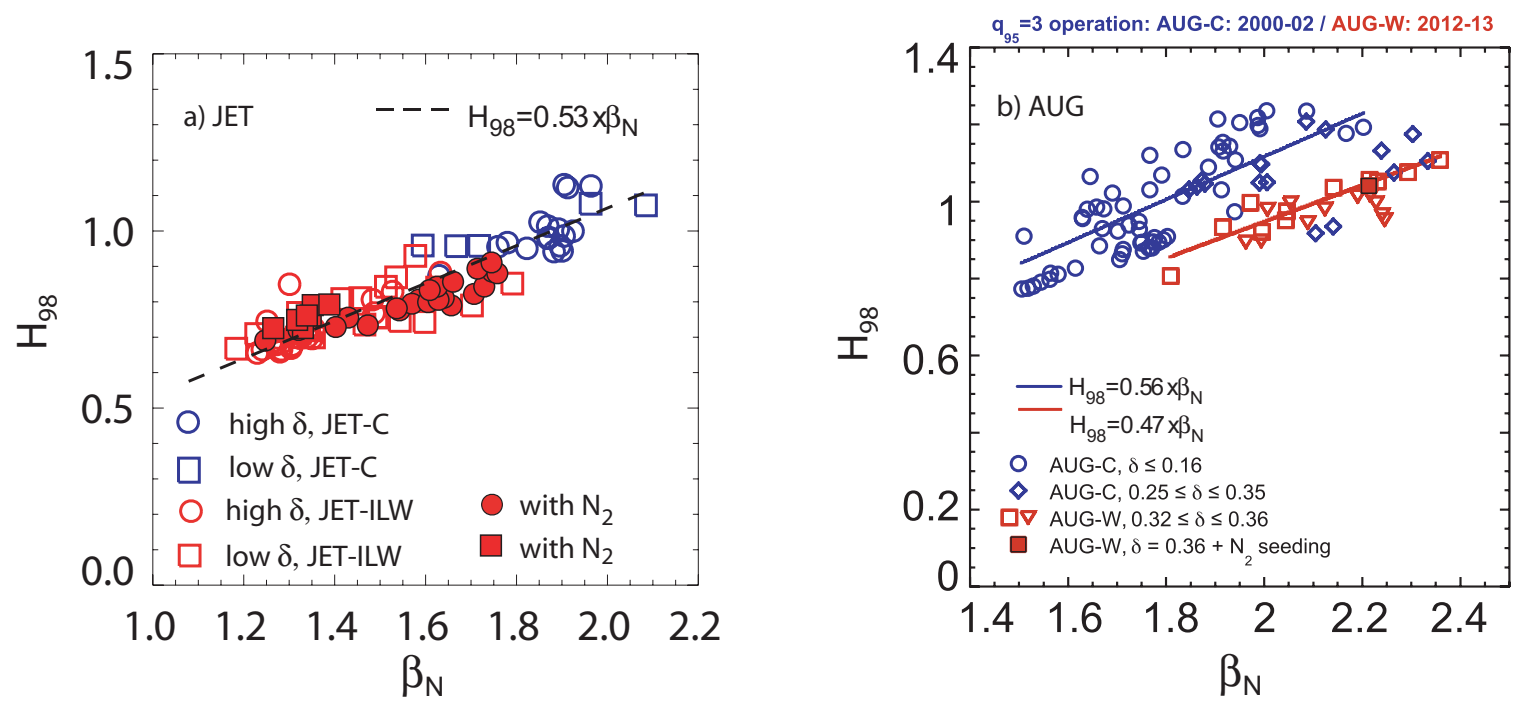

Figure 3: normalised confinement $H_{98(y, 2)}$ vs $\beta_{N}$ for a) JET and b) AUG. the hashed lines are simple linear fits to the JET and AUG datasets. The JET-ILW and JET-C data follow the same trend line. The $A U G-W$ data follow $a \sim 10 \%$ lower trend line compared to the AUG-C data.

\section{Global confinement changes due to the absence of carbon}

In addition to the change in operational requirements (operation at elevated gas fuelling) due to the presence of a metal wall, the absence of carbon as a first wall material in some cases can also affect the plasma confinement. This is evident from experiments with nitrogen seeding in AUG and JET. Nitrogen has similar ionisation energies compared to carbon and seeding of nitrogen can lead to improved confinement.

In order to show the impact of the absence of carbon, an experiment was conducted in AUG-W with $\mathrm{CD}_{4}$ seeding. In a plasma (\#27335) where the deuterium fuelling was switched off while the $\mathrm{CD}_{4}$ seeding was started, the normalised confinement was higher in the $\mathrm{CD}_{4}$ phase $\left(\mathrm{H}_{98(\mathrm{y}, 2)}=1.1\right)$ compares to the $\mathrm{D}_{2}$ phase $\left(\mathrm{H}_{98(\mathrm{y}, 2)}=1.0\right)$, as can be seen in Figure 4 . The result is not unequivocal as the $\mathrm{CD}_{4}$ seeding rate was not well calibrated. In a second pulse (\#27333) where $C_{4}$ was seeded without switching off the $\mathrm{D}_{2}$ fuelling no confinement 
improvement was found, which may be caused by over-fuelling of the plasmas. Nevertheless, this experiment provides an indication that the presence of carbon can have a beneficial effect on confinement. Future experiments will have to tune the $\mathrm{CD}_{4}$ seeding level and $\mathrm{D}_{2}$ fuelling level to obtain a clearer picture of the effect of carbon on confinement in AUG-W.

In AUG-W baseline H-mode plasmas extensive experiments with nitrogen seeding are planned for the near future. In [16] the first results of earlier experiments are presented, but remain as yet inconclusive as to whether nitrogen affects the confinement in baseline $\mathrm{H}$-mode plasmas. For AUG improved H-mode plasmas the effect of nitrogen seeding on confinement has been reported in [15]. Figure 5 shows two examples in AUG-W of improved H-mode plasmas with strong $\mathrm{D}_{2}$ fuelling at $2.2 \times 10^{22}$ electrons/s and with $\mathrm{I}_{\mathrm{p}}=1 \mathrm{MA}, \mathrm{B}_{\mathrm{t}}=2.5 \mathrm{~T}$ and $\mathrm{P}_{\mathrm{NBI}}=10 \mathrm{MW}, \mathrm{P}_{\mathrm{ICRH}}=2 \mathrm{MW}$ and $\mathrm{P}_{\mathrm{ECRH}}=2 \mathrm{MW}$. The strong $\mathrm{D}_{2}$-fuelling has affected the confinement and only $\mathrm{H} 98(\mathrm{y}, 2)=0.8$ is achieved in both cases. In Figure $5 \mathrm{a}$, from $2.5 \mathrm{~s}$ onwards nitrogen is seeded at $2 \times 10^{22}$ electrons/s in addition to the deuterium fuelling. As a result, at constant input power, the plasma stored energy is increased and $\mathrm{H}_{98(\mathrm{y}, 2)}$ rises from 0.8 to 1.2 while the normalised pressure increases from $\beta_{N}=2$ to 2.8 . In Figure $5 \mathrm{~b}$, the experiment is repeated in an attempt to keep the normalised pressure fixed, and to verify whether the confinement improvement with $\mathrm{N}_{2}$ seeding can also take place at fixed $\beta_{\mathrm{N}}$. In the experiment the NBI power is reduced from 10MW to $8.5 \mathrm{MW}$ in $\beta$-feedback mode, and only a small change in $\beta_{\mathrm{N}}=2$ to 2.4 is observed. Nevertheless, the normalised confinement is still increased from $\mathrm{H}_{98(\mathrm{y}, 2)}=0.8$ to 1.0 as soon as the nitrogen is seeded at $3 \mathrm{~s}$. Unfortunately, in this experiment the $\beta$-feedback control system did not manage to control $\beta_{\mathrm{N}}$ to remain fixed and future experiment are planned to further decouple $\beta_{\mathrm{N}}$ and $\mathrm{H}_{98(\mathrm{y}, 2)}$.

In JET-ILW nitrogen seeding was conducted in baseline $\mathrm{H}$-mode plasmas at high $(\delta=0.4)$ and low $(\delta=0.2)$ triangularity $[10,11,12]$. Figure $2 \mathrm{~b}$ shows that for a wide range of $\mathrm{D}_{2}$ fuelling from 1 to $410^{22}$ electrons/s, nitrogen seeding enhances the confinement for the high $\delta$ plasmas and approaches the confinement of the JET-C high $\delta$ data. However, a similar scan in the low $\delta$ plasmas shows that nitrogen seeding does not affect the normalised confinement in this configuration. We observe here that the different confinement properties due to plasmas shaping, as were observed in JET-C, are re-introduced with $\mathrm{N}_{2}$ seeding in JET-W.

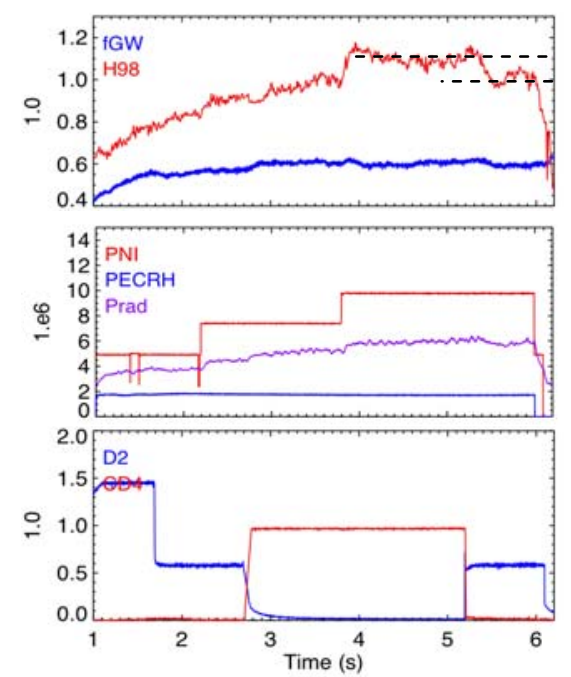

Figure 4: $\mathrm{CD}_{4}$ seeding in $A U G-W$ pulse \#27335. At 5.2 s the $C D_{4}$ seeding is switched off and the $D_{2}$ fuelling is re-introduced. This leads to a reduction in the confinement enhancement factor from $H_{98(y, 2)}=1.1$ to 1.0. 

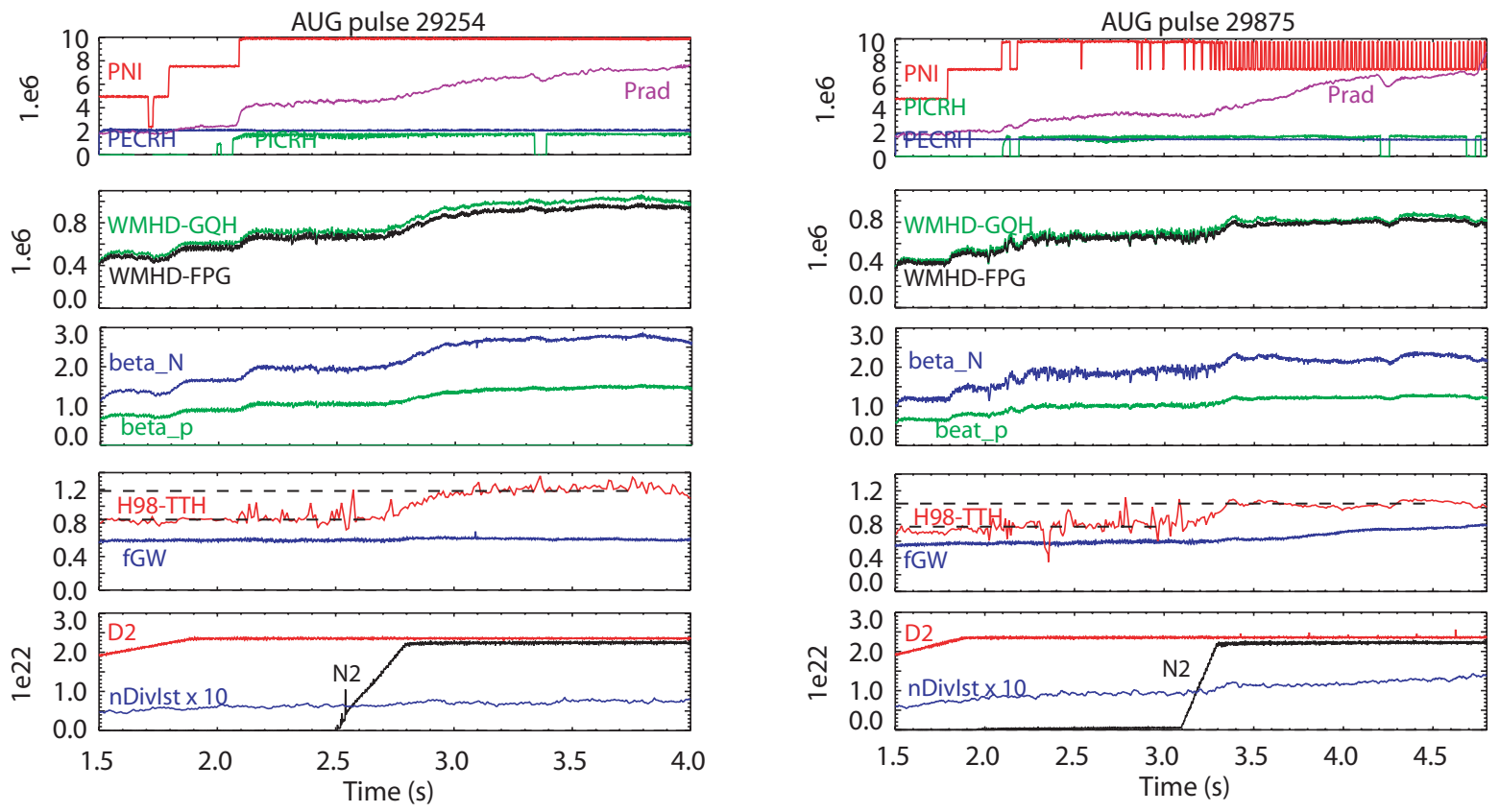

Figure 5: nitrogen seeding in AUG-W with time traces of key parameters for (left) 29254, with constant input power and (right) 29875 with $\beta$-feedback.

\section{Review of possible physics mechanisms to describe the confinement change}

\subsection{Core confinement improvement with impurity seeding}

A comparison of the linear and non-linear gyrokinetic stability analysis has been performed in [19] for AUG-W for two improved H-mode plasmas with and without $\mathrm{N}_{2}$ seeding (\#24681 and \#24282, as reported in [13]). Non-linear gyrokinetic simulation with the GENE code [20] have been conducted for these plasmas. Figure 6 shows a scan of $\mathrm{R} / \mathrm{L}_{\mathrm{Ti}}$ and it is found that the predicted modification of $\mathrm{R} / \mathrm{L}_{\mathrm{Ti}}$ at the relevant heat flux level is negligible in the case considered. At half radius $\rho_{\text {tor }}=0.5$ (Figure 6a) the critical threshold shows no upshift, whereas at $\rho_{\text {tor }}=0.8$ (Figure 6b) the critical threshold for $\mathrm{R} / \mathrm{L}_{\mathrm{Ti}}$ is higher for the seeded discharge, but the stronger profile stiffness associated with a $10-15 \%$ higher $\mathrm{T}_{\mathrm{e}}$ compensates this shift, since the ITG driven heat flux above criticality scales like $\mathrm{T}_{\mathrm{e}}^{5 / 2}$. Consistent with experimental observations in [15] the inversed gradient length $\mathrm{R} / \mathrm{LT}_{\mathrm{i}}$ is the same for both cases and no improvement in the core confinement due to $\mathrm{N}_{2}$ seeding is the result. 


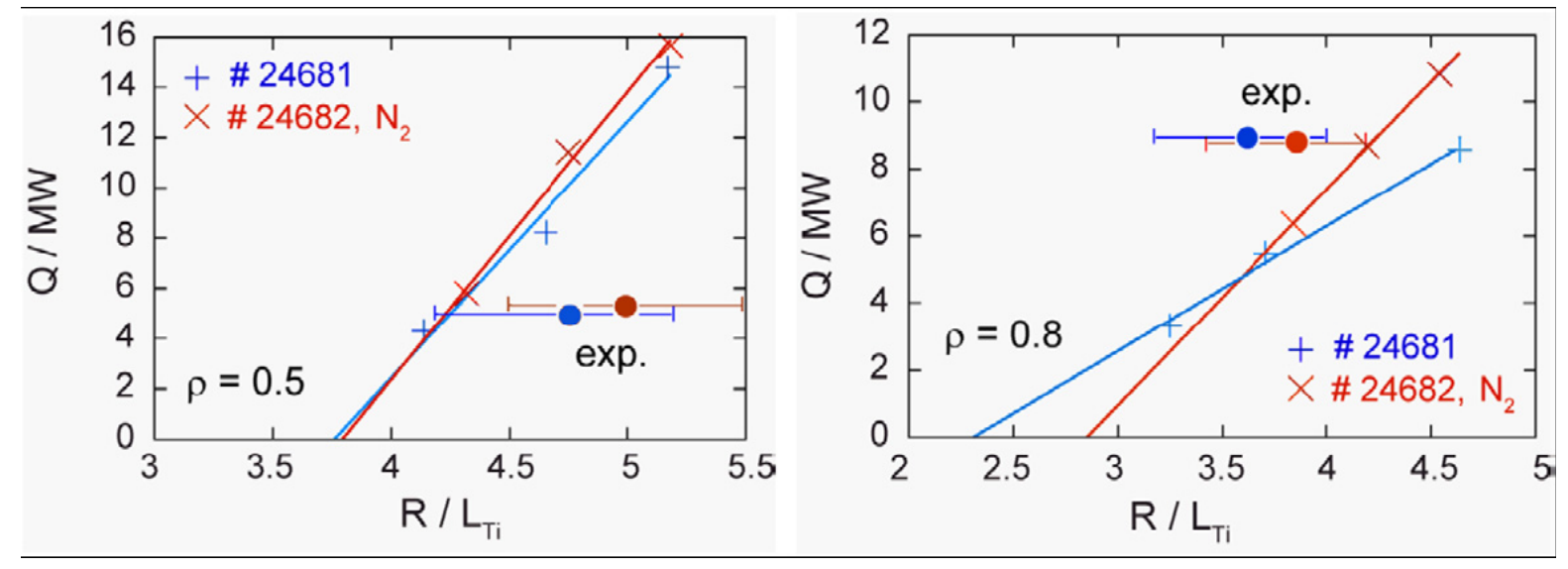

Figure 6: $R / L_{T i}$ scan with the non-linear gyrokinetic code GENE, at $\rho_{t o r}=0.5(a)$ and at $\rho_{t o r}=0.8(b)$. The full circles represent the heat flux simulated with TRANSP and the measured $R / L_{T i}$. (Figure copied from [19] with permission)

A temperature and density profile comparison (measured with the JET high resolution Thomson scattering system) is given in Figure 7 for three baseline high $\delta$ pulses in JET-C and JET ILW :1) a JET-C pulse with $2.5 \times 10^{22}$ electrons/s $D_{2}$-fuelling, $\mathrm{W}_{\mathrm{th}}=6 \mathrm{MJ}$, \#79503 2) a JET-ILW pulse with $3 \times 10^{22}$ electrons/s $\mathrm{D}_{2}$-fuelling, $\mathrm{W}_{\mathrm{th}}=4.1 \mathrm{MJ}, \# 82806$ and 3 ) a JET-ILW pulse with $2 \times 10^{22}$ electrons $/ \mathrm{s}_{2}$-fuelling and $1.5 \times 10^{22}$ electrons $/ \mathrm{s}_{2}$ seeding, $\mathrm{W}_{\mathrm{th}}=5.8 \mathrm{MJ}$, \#82817. The pulses have similar input power $\left(\mathrm{P}_{\mathrm{NBI}}=15-18 \mathrm{MW}\right)$. Figure $7 \mathrm{a} \& \mathrm{~b}$ show that the JET-ILW pulse 82806 has a lower temperature and density across the entire profile compared to the JET-C reference pulse 79503 . However, in pulse 82817 with nitrogen seeding both the temperature and the density are raised. The temperature and density profile shapes are compared in Figure $7 \mathrm{c} \& d$ by normalising the profiles to their pedestal top values. The temperature profiles across the three pulses are self-similar and show that the improvement does not come from an improved core temperature profile steepening. The density profiles shapes vary slightly, which can be ascribed to the collisionality scaling of the density peaking [21].

Figure 8 shows the temperature, density and pressure peaking for all the pulses of the JETC/JET-ILW database, as first discussed in [11]. From this comparison it is clear that the core temperature gradient lengths are not enhanced in any of the JET-C and JET-ILW pulses. If anything, the JET-ILW pulses with the poorest confinement have the strongest core temperature peaking compared to the JET-C and JET-ILW-(with $\mathrm{N}_{2}$ seeding) pulses. The density peaking in this dataset is consistent with the collisionality scaling reported in [21] which has also been observed for a wider database comparison of JET-C and JET-ILW scenarios $[6,17,18]$. The result is that across the JET-C and JET-ILW low and high $\delta$ baseline $\mathrm{H}$-mode comparison the pressure peaking shows a small variation across the entire dataset. It should be noted that only the electron temperature is presented here. However, due to the high plasma density in these plasmas, the electron-ion exchange time is much shorter than the energy confinement time and hence $\mathrm{T}_{\mathrm{i}} \sim \mathrm{T}_{\mathrm{e}}[6]$.

As a conclusion in cases where Nitrogen seeding helps recover the confinement in AUG-W (improved H-mode plasmas) and JET-ILW high triangularity baseline plasmas there is no sign of enhanced profile peaking benefitting the global confinement 

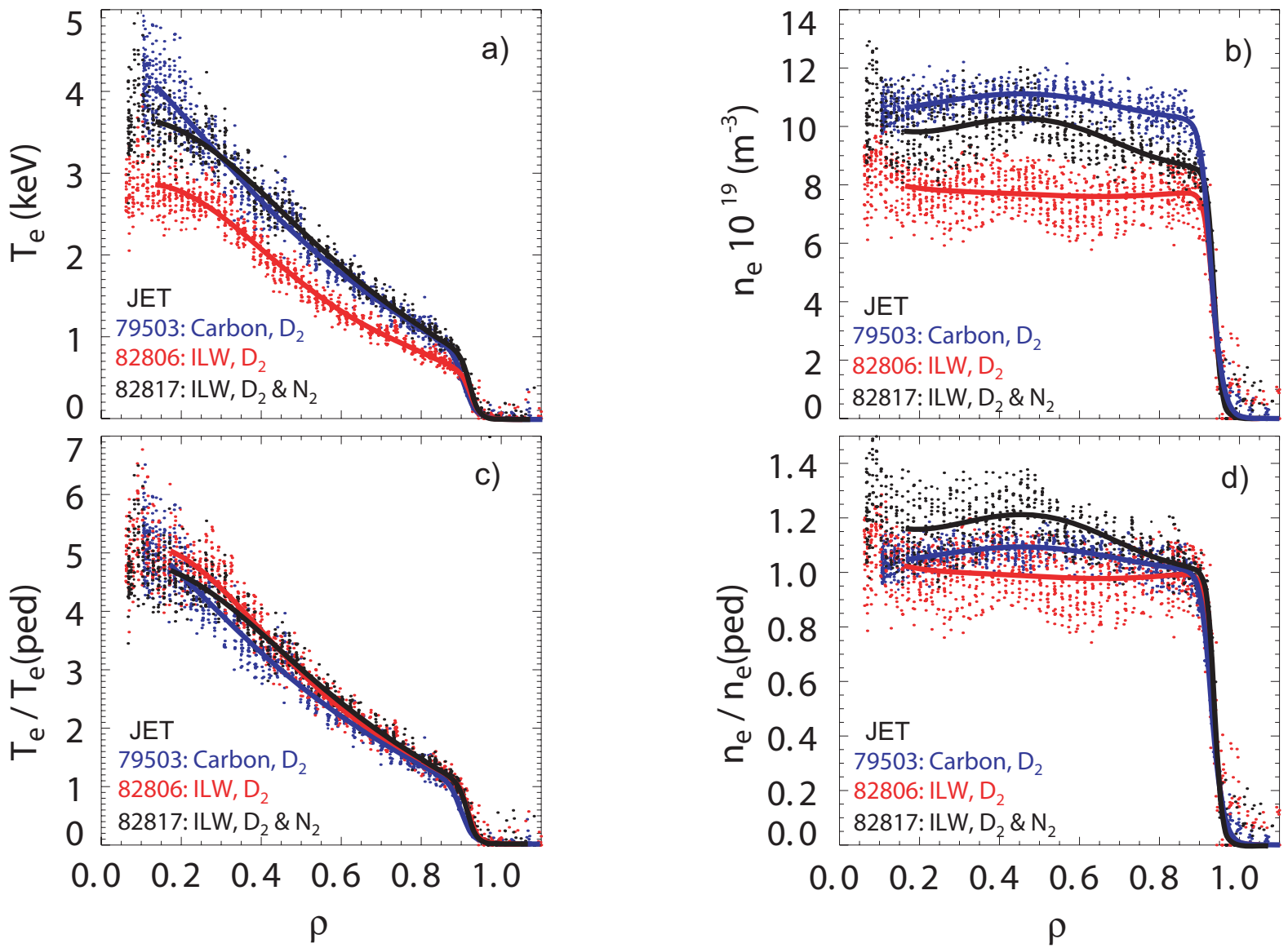

Figure 7: Profile comparison for JET-C and JET-ILW high $\delta$ baseline plasmas - see main text. a) electron temperature, b) electron density. $c \& d$ ) Normalised temperature and density.
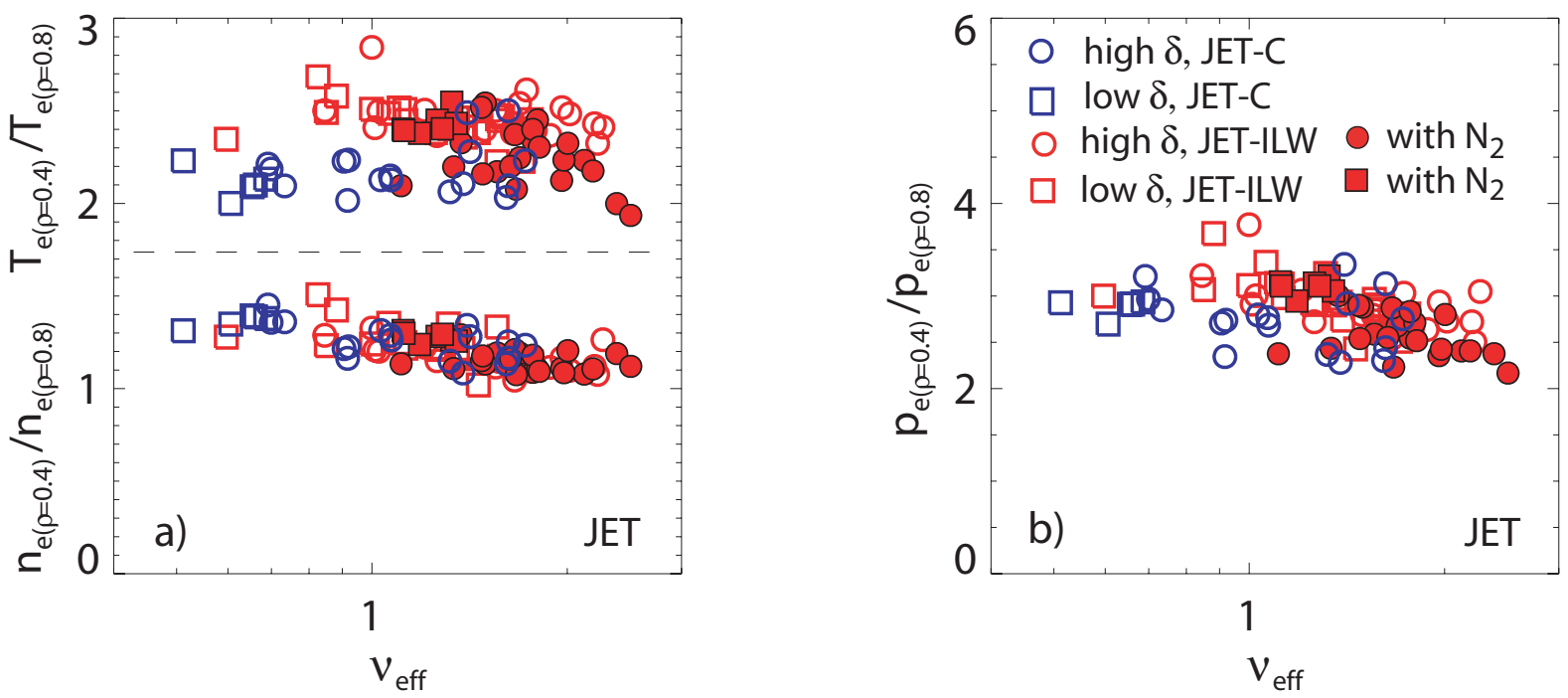

Figure 8: JET temperature, density (a) and pressure (b) profile peaking for pulse selection as in Figure 2. 


\subsection{Confinement improvement due to pedestal ion-dilution.}

The dilution of the edge ion density due to impurity seeding can lead to an increase in pedestal temperature if the total pedestal pressure is conserved. Assuming nitrogen as the main impurity (with charge $\mathrm{z}_{\mathrm{i}}=7$ ) the increase in pedestal temperature can be calculated as follows:

$$
\begin{aligned}
& p_{\text {ped }}=k \times\left(n_{e, p e d} T_{e, p e d}+n_{i, p e d} T_{i, p e d}\right)=k \times\left(n_{e, p e d}+n_{i, p e d}\right) \times T_{p e d}=C \\
& n_{i, p e d}=n_{e, p e d} \times \frac{\left(z_{i}+1\right)-z_{e f f, p e d}}{z_{i}}=n_{e, p e d} \times \frac{8-z_{e f f, p e d}}{7} \\
& T_{p e d}=\frac{p_{p e d}}{k \times\left(n_{e, p e d}+n_{i, p e d}\right)}=\frac{p_{p e d}}{k \times n_{e, p e d}} \times \frac{1}{\left(1+\frac{8-z_{\text {eff }, p e d}}{7}\right)}
\end{aligned}
$$

With $p_{\text {ped, }}, T_{\text {ped }}$ and $n_{\text {ped }}$ the pedestal pressure, temperature and density for electrons (e) and ions (i), $k$ the Boltzmann factor, $z_{i}$ the main ion charge, and $z_{\text {eff,ped }}$ the effective ion charge at the pedestal top. As an example, if the pedestal effective charge changes from $Z_{\text {eff }}=1.3$ to 4 , this would lead to an increase of $\mathrm{T}_{\text {ped }}$ by $25 \%$ with a preservation of the pedestal pressure.

In conditions of core temperature profile stiffness, an increased pedestal temperature leads to an equivalent increase of the core temperature. This can lead to a net confinement benefit if (and only if) the $Z_{\text {eff }}$ profile is hollow, as is illustrated in Figure 9 with a simulation of a flat (case 1) and hollow $Z_{\text {eff }}$ profile (case 2) assuming a circular geometry for the volume integrals of the kinetic profiles to determine the thermal stored energy. In case 1 the pedestal $Z_{\text {eff }}=1.3$ whereas in case 2 it is $Z_{\text {eff }}=4$, where at constant pressure, the latter leads to a relative increase of the pedestal temperature of $\sim 25 \%$. The hollow $Z_{\text {eff }}$ profile in case 2 then leads to an increase of the total pressure peaking and, as obtained from the profile integrals, an increase in thermal stored energy of $21 \%$.

Thus edge dilution in combination with hollow impurity-concentration profiles can lead to improved global confinement as was first suggested in [15] as the reason for the improved confinement in the $\mathrm{N}_{2}$ seeded improved H-mode plasmas. However, in order to get the $20-40 \%$ confinement improvement as observed in some of the AUG-W improved Hmodes (e.g. Figure 5) strong edge peaking of $Z_{\text {eff }}$ is required. This strong peaking of $Z_{\text {eff }}$ has not been observed in either AUG or JET experiments.

In AUG the core $Z_{\text {eff }}$ profile can be reconstructed from Bremsstrahlung measurements, utilizing the passive background measurements of spectroscopic diagnostics [22] and directly by combining the electron density and CXRS measured impurity density profiles. Figure 10 shows the $Z_{\text {eff }}$ profiles using both methods for pulse 29254 and 29875 for the unseeded and $\mathrm{N}_{2}$-seeded phase of the experiment (with $\mathrm{H}_{98(\mathrm{y}, 2)}$ changing by $40 \%$ from 0.8 to 1.2 and by $20 \%$ from 0.8 to 1.0 , respectively). Although an increase in $Z_{\text {eff }}$ from 1.3 to 2 is observed, this takes place over the entire profile rather than the required edge localised increase.

The measurement of impurity density and $Z_{\text {eff }}$ at the edge are particularly challenging, owing to the steep gradients and the sensitivity of the result to the alignment of the CXRS and electron density measurements. However, within the uncertainty of the profile alignment there is no evidence that the $Z_{\text {eff }}$ profiles is as strongly peaked in the pedestal region as is 
shown in the simulation in Figure 9 and as required to get a $\sim 20-40 \%$ confinement improvement.

The JET experiments also show no strong evidence for strong edge peaking of $Z_{\text {eff }}$. In [11] it is shown that in JET-C high $\delta$ baseline plasmas with deuterium fuelling the $Z_{\text {eff }}$ profile is rather flat, and even peaks in the core. Unfortunately no $Z_{\text {eff }}$ profiles were available for JET-ILW in [11], so no conclusions can be drawn from the role of the $Z_{\text {eff }}$ profile shape on the confinement. However, the line averaged $\left\langle\mathrm{Z}_{\mathrm{eff}}\right\rangle$ from Bremsstrahlung spectroscopy is shown to increase only slightly or even remains unchanged when nitrogen is injected. This means that no large changes in the $Z_{\text {eff }}$ profile can be justified by the line integrals of $\left\langle Z_{\text {eff }}\right\rangle$.

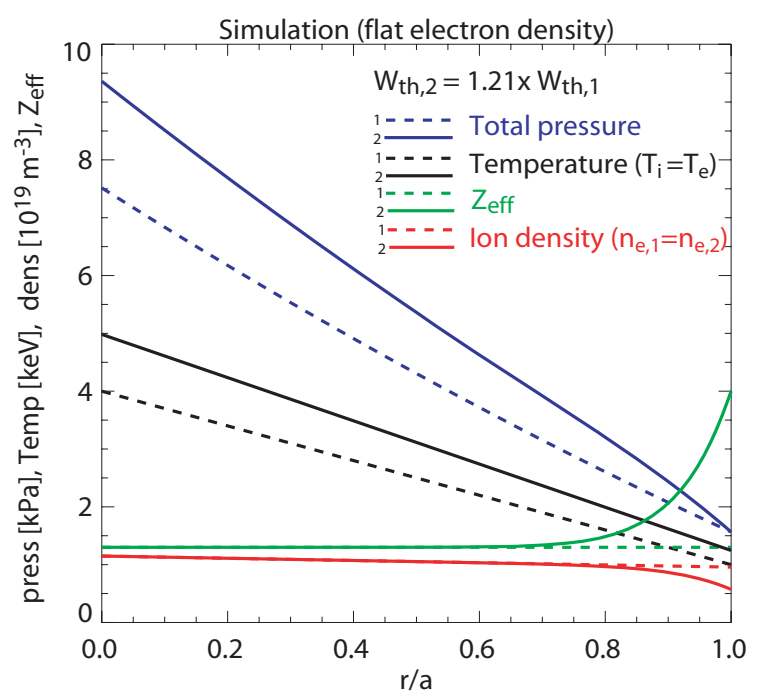

Figure 9: Simulations of the effect of a hollow Zeff profile on plasma stored energy using a circular poloidal geometry. Case 1 has a flat profile with $Z_{\text {eff }}=1.3$ and case 2 has a strong edge peaking with $Z_{\text {eff }}=4$ at the last closed flux surface falling off rapidly to $Z_{\text {eff }}=1.3$ at $r / a \leq 0.8$. The difference is stored energy using the volume integrals of the pressure profile is $21 \%$. 

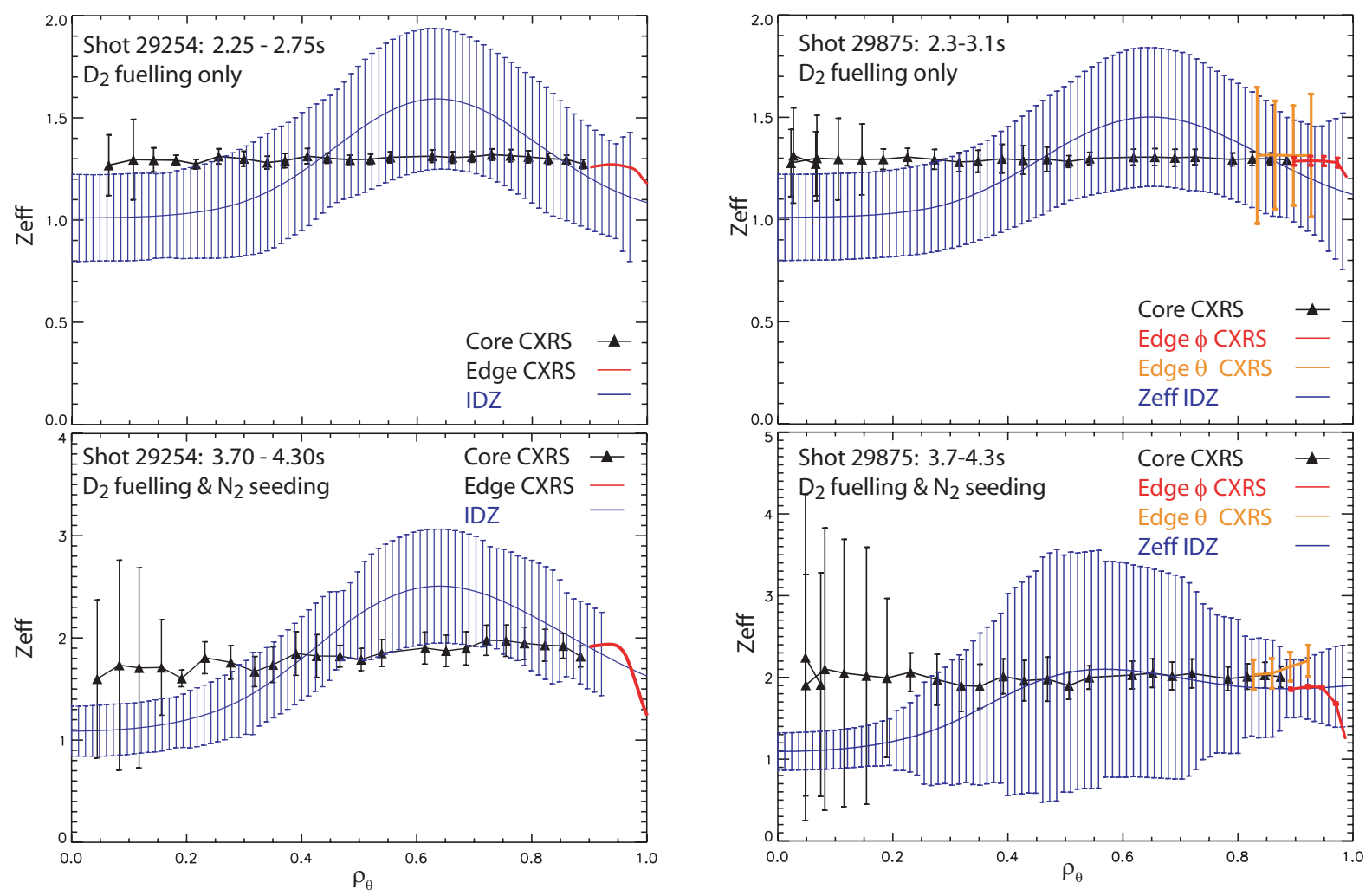

Figure 10: AUG $Z_{\text {eff }}$ profiles for pulses 29254 and 29875 during the phase (top) without $N_{2}$ seeding and the phase (bottom) with $N_{2}$ seeding from the core charge exchange diagnostic (black, edhe charge exchange diagnostics (red and orange)) and the integrated data analysis method IDZ (blue). There is no sign of strong edge peaking of $Z_{\text {eff }}$ 


\subsection{Change in edge pedestal structure and stability}

In both AUG-W [15] and JET-ILW [10, 11] the reduction of confinement going from a carbon to a metal wall as well as for cases with a recovery of the confinement through impurity seeding are generally due to changes in the pedestal pressure (Figure 11, Figure 12) in combination with core profile stiffness (section 4.1).

In AUG baseline $\mathrm{H}$-mode plasmas, nitrogen seeding experiment have not yet extensively been conducted. A detailed pedestal structure study across a broad fuelling and nitrogen seeding scan in baseline H-mode plasmas is planned for the next experimental campaign. However in the improved H-mode database discussed in [15] the effect of $\mathrm{N}_{2}$ is clearly seen on the pedestal pressure; when nitrogen is seeded the pedestal temperature increases, (Figure 11) whereas the density remains unchanged. It has also been demonstrated that the temperature pedestal widens when nitrogen is seeded [15].

In JET-ILW baseline plasmas with $\mathrm{D}_{2}$-fuelling the pedestal pressure is much reduced compared to the JET-C baseline plasmas, Figure 12 . This is especially true for high $\delta$ plasmas $[10,11$ 17, 18]. The JET-ILW high triangularity plasmas cover a similar density range compared to the JET-C plasmas, but have a pedestal temperature reduced by as much as $40 \%$ compared to JET-C. When nitrogen is seeded the pedestal pressure in JET-ILW high $\delta$ plasmas is increased and approaches that of the JET-C high $\delta$ plasmas, through an increase of both pedestal temperature and density $[10,11,12]$. The same increase in pedestal pressure is not observed in JET-ILW low $\delta$ plasmas. In this section we study the changes in the pedestal structure (pedestal width, gradient and height) in the high triangularity ELMy H-mode plasmas as a result of the changeover from a carbon to a metal wall and due to nitrogen seeding.

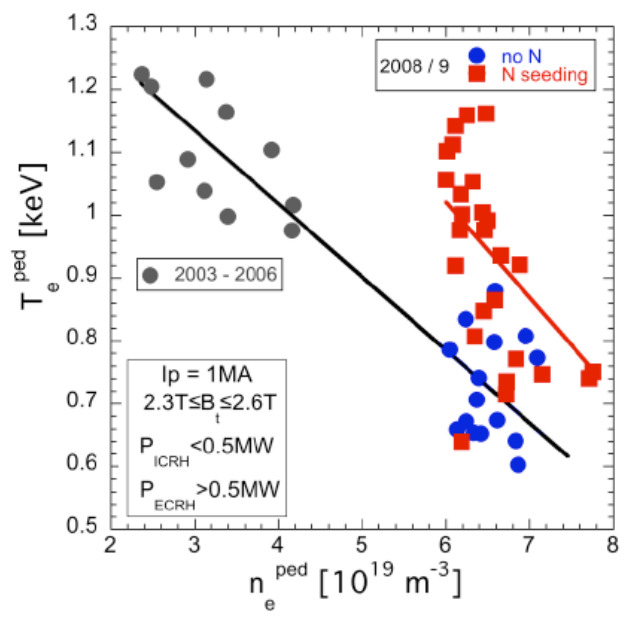

Figure 11: Electron temperature and density values close to the pedestal top $\left(\rho_{\text {pol }}=0.9\right)$. All results of the 2008/9 all- $W$ campaigns are at densities above $5.5 \times 10^{19} \mathrm{~m}^{-3}$ and well separated from the lower ones of previous campaigns (grey symbols). Puffing of $\mathrm{N}_{2}$ (red symbols) does not change the typical pedestal density of improved H-modes, but increases the temperature. This indicates an increase of the electron pressure at the pedestal top

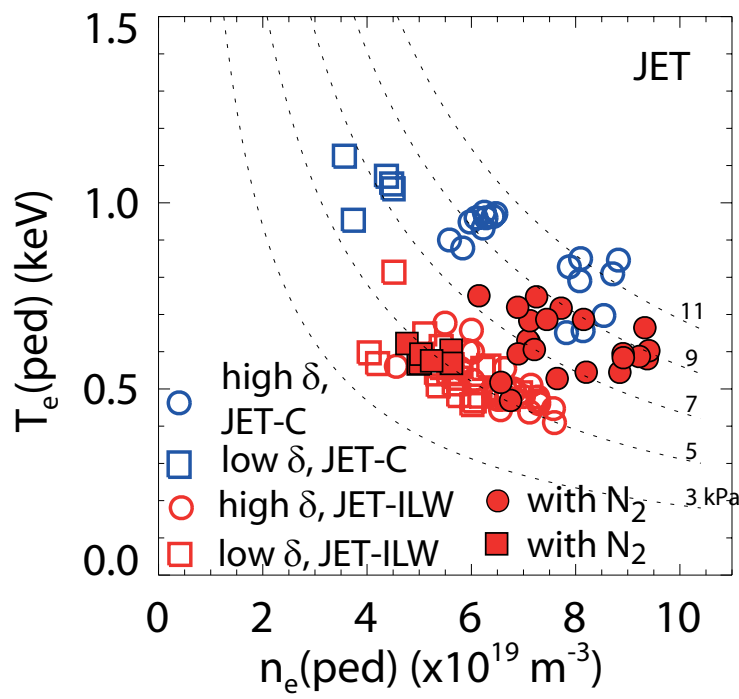

Figure 12 Pedestal $T_{e}$ and $n_{e}$ diagram for JET low and high triangularity baseline plasmas. The reduction in pedestal pressure is larger for the JET-ILW (blue) high triangularity plasmas (open circles) than for the low triangularity plasmas (open squares) compared to the JET-C baseline plasmas (blue). Nitrogen seeding partly recovers the pedestal pressure for the JET-ILW high triangularity plasmas (closed circles), but does not enhance the pressure in the JET-ILW low triangularity plasmas (closed squares) 
In JET the pedestal structure is measured in the outer mid-plane with the High Resolution Thomson scattering system (HRTS) [23]. In order to improve the accuracy of the pedestal structure measurement multiple profiles of $T_{e}$ and $n_{e}$ are collected during a few seconds steady phase of the plasma discharge. Profile selection is synchronised with respect to the individual ELM cycle and for the discussion here profiles are selected during the last $30 \%$ of the ELM cycle, i.e. these profiles are representative of the pre-ELM phase. Figure 13 shows examples from two plasmas. The pedestal width, height and gradient are obtained from modified tangent hyperbolic fits as in [24] and as shown in the figure.
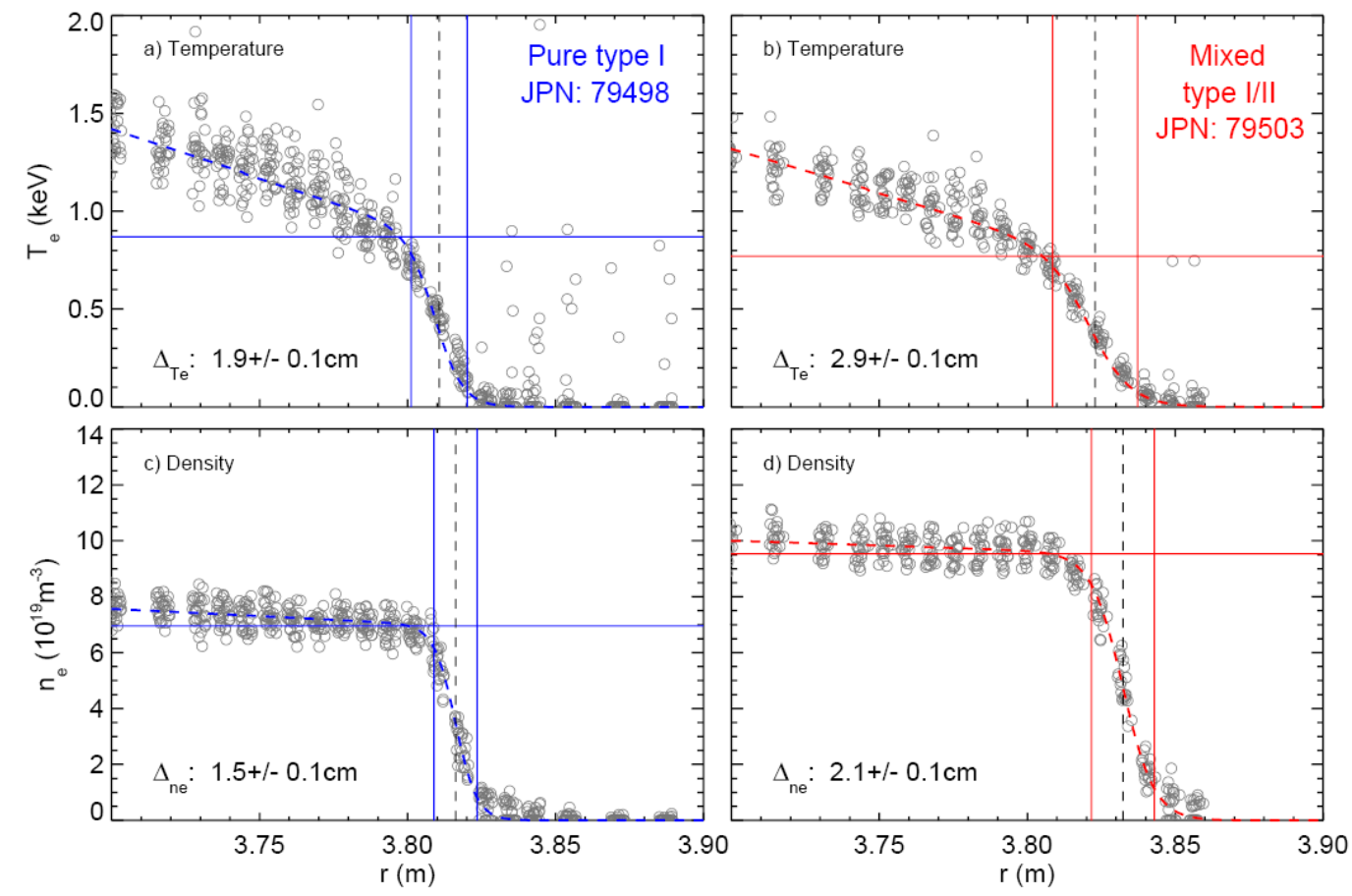

Figure 13: Example profiles for two plasmas in JET-C with low and high fuelling from [8]

In JET-C the pedestal structure in high triangularity baseline plasmas was studied in [8]. It was found (Figure 13) that both the temperature and density pedestal widen when the fuelling is increased from $0.2 \times 10^{22}$ to $2.5 \times 10^{22}$ electrons/s; the temperature pedestal widens from $\Delta \mathrm{T}_{\mathrm{e}}=1.9 \pm 0.1 \mathrm{~cm}$ to $2.9 \pm 0.1 \mathrm{~cm}$, and the density pedestal from $\Delta \mathrm{n}_{\mathrm{e}}=1.5 \pm 0.1$ to $2.1 \pm 0.1 \mathrm{~cm}$. The HRTS system was upgraded from a spatial resolution of $2 \mathrm{~cm}$ to a spatial resolution of $1 \mathrm{~cm}[25,26]$ towards the end of JET operation with the carbon wall, and for this reason no more extensive pedestal analysis is available for this time period. Figure 14a, c,d show that in JET-C the widening of the pedestal at increased fuelling together with only a moderate decrease in the pressure gradient leads to an increased pedestal pressure and an improved pedestal confinement (here the pedestal width is expressed in normalised poloidal flux space to enable comparison with the EPED model). In [8] it was not clear whether this widening of the edge barrier is a result of the fuelling per se or is linked to the observed transition from a pure Type I ELM $\left(\mathrm{f}_{\mathrm{ELM}}=20 \mathrm{~Hz}, 79498\right)$ to a mixed Type I/II ELM regime $\left(\mathrm{f}_{\mathrm{ELM}}=7 \mathrm{~Hz}, 79503\right)$.

Figure $14 \mathrm{a} \& \mathrm{c}$ show that across a $\mathrm{D}_{2}$-fuelling scan at constant input power $\mathrm{P}_{\mathrm{NBI}}=15 \mathrm{MW}$ in the JET-ILW high triangularity experiments the pedestal also widens but is accompanied by a strong decrease in the pedestal pressure gradient. As a result there is no change in the obtained pedestal pressure, Figure $14 \mathrm{~d}$, and hence no increase in global confinement is seen as in the JET-C $\mathrm{D}_{2}$ fuelling experiment. Note however, that in this JET- 
ILW dataset also no transition from Type I to mixed type I/II ELMs occurs, as no reduction in ELM frequency is observed in the gas scan with $\mathrm{f}_{\mathrm{ELM}}>20 \mathrm{~Hz}$.

In the JET-ILW $\mathrm{N}_{2}$-seeding scan with fixed $\mathrm{D}_{2}$ fuelling at $1 \times 10^{22}$ electrons/s, we again see the pedestal widening in Figure 14e, but in this case the pedestal gradient does not degrade or even increases (Figure 14g). The combination of the two leads to an increase in pedestal top pressure as seen in Figure 14h. In this data-set we do observe a change in ELM regime; in pulse 82585 the ELM frequency is $20 \mathrm{~Hz}$ which decreases to $5 \mathrm{~Hz}$ for pulse 82814 . This lowering of the ELM frequency resembles the transition from what was described in $[4,8,9]$ from a pure Type I to a mixed type I/II ELM regime in JET-C.

In order to get improved pedestal confinement it is necessary to increase the pedestal width and gradient simultaneously. The JET experiments show that both are seen to vary independently; in JET-ILW the pedestal width increases with increased $\mathrm{D}_{2}$ fuelling, but the pedestal gradient reduces, leading to no change in pedestal pressure. In the JET-ILW $\mathrm{N}_{2-}$ seeding and the JET-C $\mathrm{D}_{2}$-fuelling experiments, the pedestal width increases while the gradient shows little change, which leads to an improved pedestal pressure. We try to understand this variation in a comparison with the predictive pedestal model EPED.

The EPED model is developed to predict the pressure pedestal structure. The inputs to the model are eight scalar parameters, $\mathrm{B}_{\mathrm{t}}(\mathrm{T})$ the toroidal magnetic field, $\mathrm{I}_{\mathrm{p}}(\mathrm{MA})$ the plasma current, $\mathrm{R}(\mathrm{m})$ the major radius, $\mathrm{a}(\mathrm{m})$ the minor radius, $\delta$ the triangularity , $\kappa$ the elongation, $\mathrm{n}_{\mathrm{e}, \mathrm{ped}}\left(10^{19} \mathrm{~m}^{-3}\right)$ the pedestal density and $\beta_{\mathrm{N}, \text { global }}$ the global Troyon normalised pressure. EPED assumes the pedestal pressure will rise until constrained by the onset of two key instabilities, the Peeling Ballooning (PB) and Kinetic Ballooning (KB) modes. These instabilities provide two calculable constraints which when combined can be solved for the two unknowns, the pedestal height and width [27, 28, 29]. The PB limit is evaluated by means an ideal MHD stability code ELITE [30, 31], which gives a calculated dependence of pedestal pressure and pedestal width. The KB modes limit the pressure gradient in a more local sense; the pedestal can grow consistently until the PB limit is reached at which point an ELM occurs. In the first version of EPED (EPED1) used here, the pedestal width is limited by the KB mode as $\Delta \sim \mathrm{p}^{1 / 2}$ [27].

Figure $14 \mathrm{~b} \& \mathrm{f}$ show the EPED predictions of the pedestal width and in Figure $14 \mathrm{~d} \& \mathrm{~h}$ a comparison of the measured pedestal pressure and the EPED pedestal pressure prediction. EPED1 gives a quantitatively good prediction of the pedestal width and height of the low fuelling pulses in JET-C (79498 and 79499, [8]) and JET-ILW (82585 and 82586). Also in general it predicts the pedestal height of the JET-C and JET-ILW baseline plasmas within the $\pm 20 \%$ model accuracy. For the JET-ILW $\mathrm{D}_{2}$ fuelling scan (Figure 14) $\mathrm{d}$ the EPED prediction of the pedestal pressure is within $5 \%$ of the measurements, but the variation of the pedestal width is not captured by the model (Figure $14 \mathrm{a} \& \mathrm{~b}$ ); the width increases in the experiment and remains constant in the EPED1 prediction. The largest deviations are observed for the cases where in the experiment the pedestal widens and the pressure gradient is only moderately affected. This is the case for the high $\mathrm{D}_{2}$-fuelling JET-C plasma (79503, [8]) and the in the JET-ILW N $\mathrm{N}_{2}$ seeding scan in Figure $14 \mathrm{e}-\mathrm{h}$. 

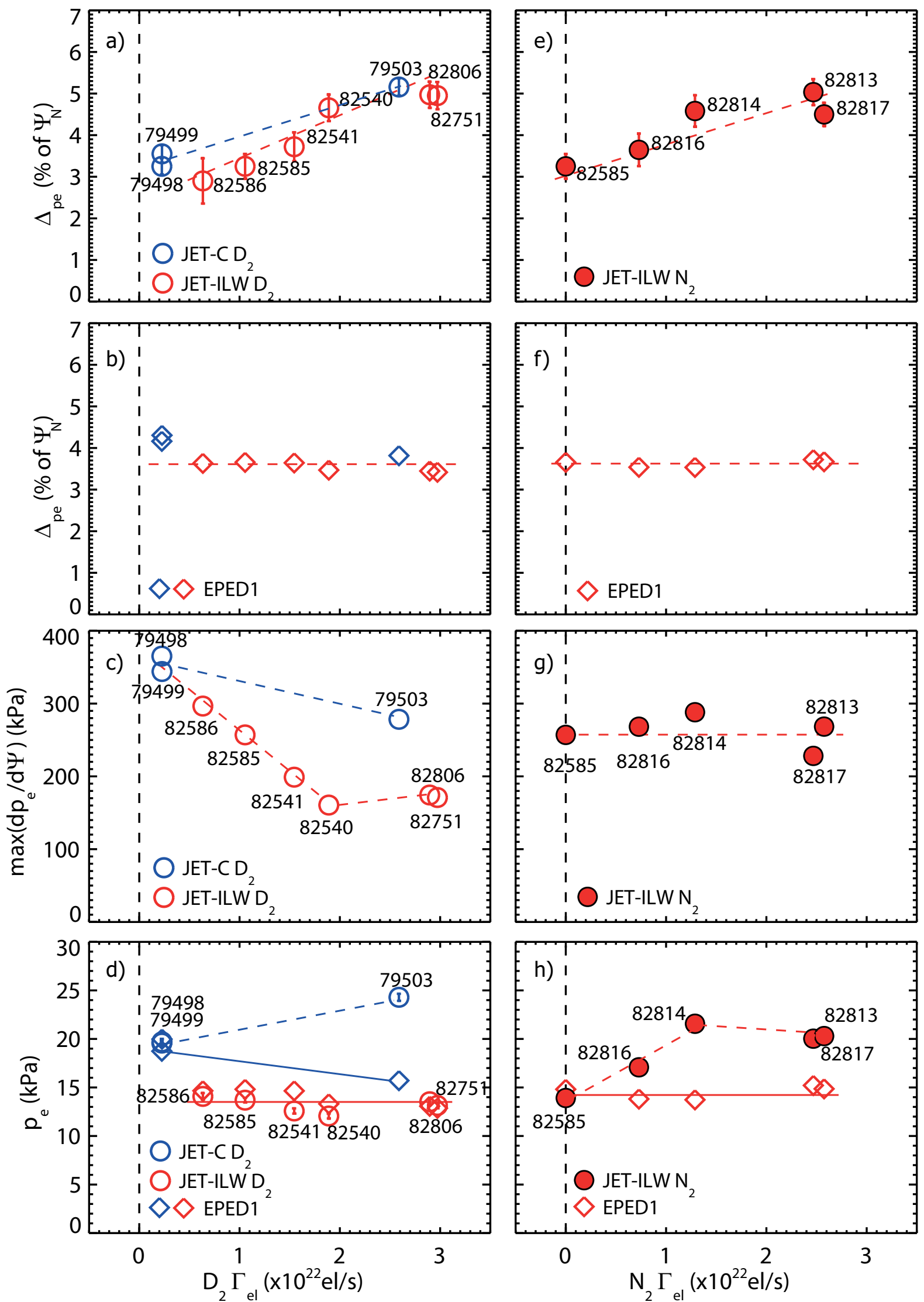

Figure 14: Pedestal structure and EPED predictions as a function of $D_{2}$ fuelling for JET-C and JETILW (a-d) and $N_{2}$ seeding for JET-ILW (d-h) 
All the plasmas in this comparison are in Type I ELMy H-mode. From this observation we would expect that the pedestal stability would be consistent with the PB model. Figure 15 shows the PB stability boundary from the ELITE code as calculated for all the JET pulses in Figure 14, as well as the experimental normalised pressure gradient $\alpha_{\max }$ and edge current $\left\langle\mathrm{j}_{\phi}\right\rangle_{\max }$ derived from the bootstrap current [32] and the fits to the pre-ELM pedestal profiles. The starting point at low $\mathrm{D}_{2}$ fuelling $\left(82585\right.$, at $1 \times 10^{22}$ electrons/s) shows the experimental point being marginally stable or just crossing the ballooning stability boundary. At lower fuelling level $\left(82586\right.$, at $0.6 \times 10^{22}$ electrons/s) the experimental point lies in the unstable region, whereas at higher fuelling (e.g. 82540 , at $1.9 \times 10^{22}$ electrons/s) the experimental points lie in the stable region of the PB stability diagram. When the nitrogen seeding is increased, the measured pedestal profile widens, which causes the ballooning boundary to shift to lower pressure gradient $\alpha_{\max }$. However the experimental point (82814) has a somewhat increased pressure gradient well into the unstable PB region compared the reference (82585).

The comparison of the JET data with the EPED predictive pedestal code and the ELITE ideal MHD calculation shows a complex variation of the pedestal width and gradient across the fuelling and seeding scans presented here. This variation is as yet not well understood and provides a challenge for pedestal modelling.
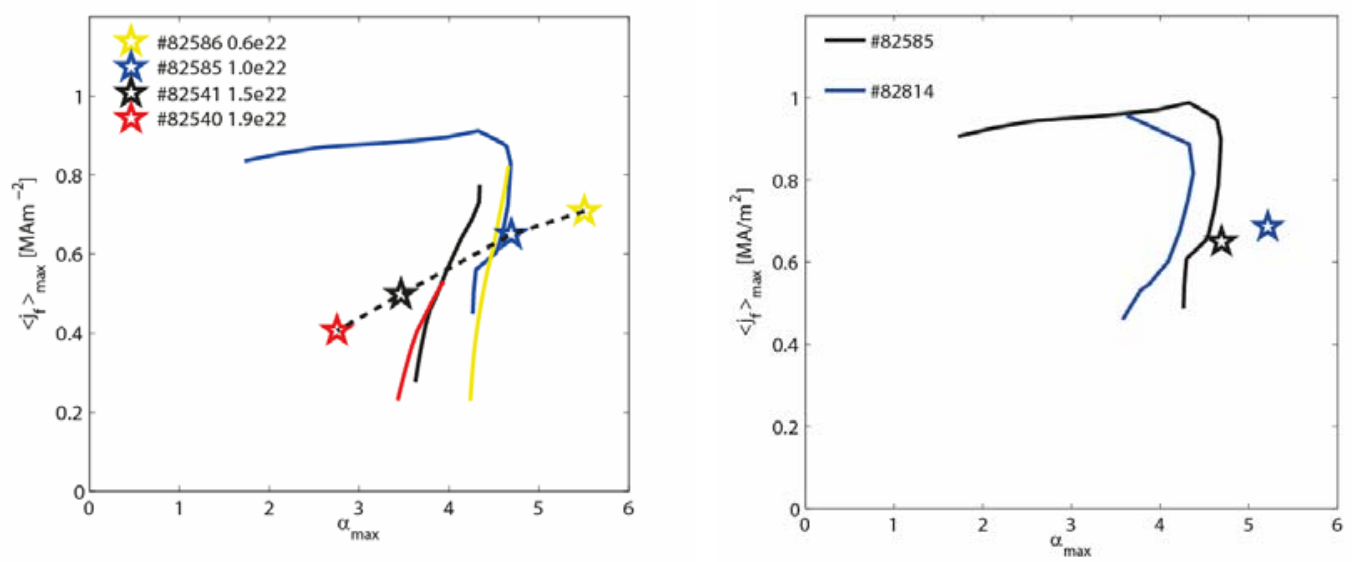

Figure 15: Peeling Ballooning stability calculations for the JET plasmas for selected cases in Figure 14 using the experimental pedestal profile fits of $T_{e}$ and $n_{e}$ and line averaged $Z_{\text {eff }}$ measurements from Bremsstrahlung. The ballooning stability boundary shifts to lower pressure gradients when the experimental pedestal profiles widen. For the $D_{2}$ fuelling scan, the lowest fuelling pulse 82586 has the narrowest pedestal and highest pressure gradient (Figure 14). This experimental point lies in the unstable PB region. When the fuelling is increased for 82858, 82541 and 82540, the pressure gradient is reduced and the experimental points move from unstable to stable with respect to the calculated PB stability boundary. In the nitrogen seeded pulse 82814 the pedestals widens and shows a somewhat higher pressure gradient compared to the reference 82585. However the PB stability boundary has moved to a lower pressure gradient and as a result the experimental point lies in the unstable region of the $P B$ stability diagram.

\section{Discussion and conclusion}

This paper reviews the impact of a metal wall on confinement in JET and AUG. The clearest difference seen between the full carbon AUG and JET and full metal devices AUG-W and JET-ILW is the loss of the well performing zero gas flux, low recycling scenario going from $\mathrm{C}$ to $\mathrm{W}$ or $\mathrm{Be} / \mathrm{W}$. The confinement loss is not found to be equal for all scenarios for each of the devices. Most notably in the JET high triangularity baseline plasmas an additional loss in 
confinement is found. In JET-C the high triangularity had a better normalised confinement than the low triangularity baseline H-mode scenario for a given Greenwald density fraction $\mathrm{n} / \mathrm{n}_{\mathrm{gw}}$. In addition the scenario could be fuelled to high densities of $\mathrm{n} / \mathrm{n}_{\mathrm{gw}} \sim 1$ and maintain good confinement. In JET-ILW however, the confinement of this scenario has decreased and resembles that of the low triangularity baseline plasmas. The confinement loss in the JETILW high triangularity scenario can be partly recovered by means nitrogen seeding. In AUG$\mathrm{W}$, the high triangularity scenario can achieve good normalised confinement of $\mathrm{H}_{98(\mathrm{y}, 2)} \sim 1$ at $\beta_{\mathrm{N}}>2$ [16] and does not seem to suffer from the same confinement loss as the JET scenario. In addition, preliminary tests show that no confinement improvement is found here with nitrogen seeding. However, in AUG-W a confinement loss is experienced in improved Hmode plasmas [15] and here nitrogen seeding has been found to recover the confinement compared to AUG-C. It therefore seems that the extent to which confinement is impacted by a metal wall in AUG and JET is variable, and that in case of a loss in confinement it can be recovered by means of nitrogen seeding. In scenarios with no or only a limited confinement loss in the metal wall devices seeding nitrogen does not seem to affect the confinement significantly indicating that nitrogen can only help recover the confinement that was lost and cannot lead to improved confinement in comparison to similar scenarios in the carbon wall devices.

Three potential mechanisms have been discussed that potentially can play a role in describing the confinement behaviour in these metal wall devices. Firstly, for AUG-W it has been demonstrated by means of nonlinear gyrokinetic simulations that nitrogen does not significantly alter the core ion temperature gradient turbulence onset and hence does not alter the core critical temperature gradient length. This is consistent with experimental observations in both AUG and JET which show that the core temperature gradient lengths remain unchanged across a fuelling and seeding scan in both AUG-W and JET-ILW, and in the latter case also in a comparison with JET-C H-mode plasmas.

Secondly, it has been demonstrated that the pedestal ion-dilution by means of nitrogen seeding cannot fully account for the confinement improvement with nitrogen seeding in AUG-W and JET-ILW. This mechanism requires strong edge peaking of the impurity concentration, which has not been observed in AUG and JET.

Thirdly, it has been shown that $\mathrm{D}_{2}$-fuelling and $\mathrm{N}_{2}$-seeding can affect the pedestal pressure due to changes in the pedestal width and gradient. In JET-C high triangularity plasmas the pedestal width increases while the pressure gradient is only modestly reduced in a $\mathrm{D}_{2}$-fuelling scan. As a result the pedestal pressure is enhanced in the higher fuelling plasmas, and the global stored energy is increased in combination with core profile stiffness. This describes how in JET-C this scenario could maintain good confinement at high fuelling levels. In JET-ILW the pedestal is also seen to widen in a $\mathrm{D}_{2}$-fuelling scan. Nevertheless, the pressure gradient is strongly reduced at the same time as the pedestal widens, and this does not lead to an increase in the pedestal top pressure, and hence does not lead to a confinement improvement. With nitrogen seeding in JET-ILW, we see a simultaneous increase in the pedestal width and only a small change in the pedestal gradient, which results in an increase in the pedestal pressure. In a comparison with EPED and ideal MHD stability analysis we find that these observations require deviations from both the ideal MHD stability limit as well as changes to the kinetic ballooning stability that are as yet not understood. Both the widening of the pedestal with gas fuelling and seeding $\left(\mathrm{D}_{2}\right.$ and $\left.\mathrm{N}_{2}\right)$ as well as the steepening of the pedestal pressure gradient in the JET-C $\mathrm{D}_{2}$-fuelling plasmas and the JET-ILW $\mathrm{N}_{2-}$ seeding plasmas require further study. The steepening of the pressure gradient at a wide pedestal barrier is correlated with the transition from type I to mixed Type I/II ELMs, which could give an important direction to the physics studies. In AUG-W improved H-mode plasmas, nitrogen seeding is also seen to increase the pedestal pressure and affect the pedestal 
structure. The variable impact of a metal wall and nitrogen seeding on scenarios in JET and AUG is consistent with its possible influence on edge stability and the pedestal structure. The role of pedestal stability on confinement is complex and it is to be expected that machine size, impurity content, pedestal temperature and edge current affect the extent as to how much the achieved pedestal pressure is affected. Detailed studies on the role of plasma shaping, impurity seeding, and scenario type in these metal-wall devices are planned to for the upcoming campaigns.

In summary, the confinement in these two metal devices is affected by changes in the obtainable pedestal pressure. In combination with core profile stiffness this leads to the changes in confinement observed. This means that for a given input power, generally a lower pedestal pressure and hence a lower plasma confinement and normalised pressure is observed in both devices without nitrogen seeding. For a given input power generally a lower $\beta_{N}$ is obtained in the JET-ILW compared to JET-C baseline H-mode plasmas. Together with the strong coupling found between $\beta_{\mathrm{N}}$ and $\mathrm{H}_{98(\mathrm{y}, 2)}$, Figure 3, this finding may potentially affect the achievable baseline H-mode plasma confinement in ITER with limited input power capability. With nitrogen seeding, higher values of $\beta_{\mathrm{N}}$ can be achieved for a given input power.

We interpret the results presented here nevertheless in favour of a start-up in ITER with an all-W divertor, since it is uncertain that an ITER scenario developed with low power in a $\mathrm{C}$ divertor can be transferred to an all-W ITER divertor. The uncertainty is obvious from the unexplained empirical behaviour shown here, and a clear recommendation is to work on the physics behind these observations. But with the present understanding, and the fact that $\mathrm{C}$ is not allowed in a nuclear phase, starting with $\mathrm{W}$ has lower risk regarding H-mode quality for the nuclear phase. 
${ }^{1}$ S. Brezinszek et al., 'Fuel Retention Studies with the ITER-like Wall in JET', 24th IAEA Fusion Energy Conferenc, San Diego, USA, 2012 and submitted to nuclear fusion

${ }^{2}$ R Neu et al 2009 Phys. Scr. 2009014038 doi:10.1088/0031-8949/2009/T138/014038

${ }^{3}$ G. Matthews et al, Physica Scripta, vol. T128, pp. 137-143, 2007.

${ }^{4}$ G. Saibene et al, 2002 Plasma Phys. Control. Fusion 441769

${ }^{5}$ ITER Physics basis, Nuclear Fusion 39 No 12 (December 1999) 2175-2249

${ }^{6}$ M.N.A. Beurskens et al, Nuclear Fusion, vol. 53, no. 1, p. 013001, 2013.

${ }^{7}$ J. Stober et al Plasma Phys. Control. Fusion 42 (2000) A211-A216

${ }^{8}$ M. Leyland, Nuclear fusion, accepted for publication

${ }^{9}$ C. Giroud et al, Nucl. Fusion 52 (2012) 063022 (22pp) doi:10.1088/0029-5515/52/6/063022

${ }^{10} \mathrm{C}$ Giroud et al, "Nitrogen seeding for heat load control in jet elmy h-mode plasmas and its compatibility with ilw materials," in Proc. "24th IAEA Fusion Energy Conference", San Diego, USA, 2012, pp. EX/P5-30.

${ }^{11} \mathrm{C}$ Giroud et al, Nuclear fusion, submitted

${ }^{12}$ G. Maddison et al, 'Contrasting H-mode behaviour with deuterium fuelling and nitrogen seeding in the all-carbon and metallic versions of JET', Submitted to Nuclear Fusion

${ }^{13}$ O. Gruber et al., Physical Review Letters 83, 1787 (1999)

${ }^{14}$ A. Staebler et al., Nuclear Fusion 45, 617 (2005).

${ }^{15}$ Schweinzer J et al 2011 Nucl. Fusion 51113003

${ }^{16}$ J. Schweinzer et al, 'Demonstration of the ITER Baseline Scenario on ASDEX Upgrade', 40th European Physical Society Conference on Plasma Physics, Espoo, Finland, 1st - 5th July $2013, \underline{\mathrm{P} 2.134}$

${ }^{17}$ M.N.A. Beurskens et al, "L-H power threshold, pedestal stability and confinement in jet with a metallic wall," in submitted to Nuclear Fusion and Proc. "24th IAEA Fusion Energy Conference", San Diego, USA, Vienna, 2012, pp. EX/P7-20.

${ }^{18}$ M.N.A. Beurskens et al, submitted to Nuclear Fusion

19 Tardini G et al 2013 Plasma Phys. Control. Fusion 55015010

${ }^{20}$ Jenko F et al 2000 Phys. Plasmas 71904

${ }^{21}$ C. Angioni et al, Physics of Plasmas 16, 060702 (2009), doi: 10.1063/1.3155498 
${ }^{22}$ S K Rathgeber et al, Plasma Physics and Controlled Fusion 52, 9 (2010), 095008

${ }^{23}$ R. Pasqualotto et al, Rev. Sci. Instrum.75, Number 10 October 2004

${ }^{24}$ R. J. Groebner et al., Plasma Phys. Controlled Fusion 44, A265 (2002).

${ }^{25}$ M.N.A. Beurskens et al, Phys. Plasmas 18, 056120 (2011)

${ }^{26}$ L. Frassinetti et al, Rev. Sci. Instrum. 83, 013506 (2012); doi: 10.1063/1.3673467

${ }^{27}$ Snyder P.B., et al., 2009. Development and validation of a predictive model for the pedestal height. Physics of Plasmas, 16:056118.

${ }^{28}$ Snyder P.B., et al., 2011. A first principles predictive model of the pedestal height and width: development, testing and ITER optimization with the EPED model. Nuclear fusion, $51: 103016$.

${ }^{29}$ Snyder, P.B. et al., 2012. The EPED pedestal model and edge localized mode suppressed regimes: Studies of quiescent $\mathrm{H}$-mode and development of a model for edge localized mode suppression via resonant magnetic perturbations. Phys. Plasmas, 19:056115.

${ }^{30}$ Wilson H.R., et al., 2002. Numerical studies of edge localized instabilities in tokamaks. Physics of Plasmas, 9:4.

${ }^{31}$ Snyder P.B. et al., 2002. Edge localized modes and the pedestal: A model based on coupled peeling-ballooning modes. Physics of Plasmas, 9:5.

${ }^{32}$ Sauter O, Angioni C and Lin-Liu Y R 1999 Phys. Plasmas 62834 